\title{
SLOPE OF SIDES OF CORNEAL GRAFTS AND RECIPIENT BEDS*
}

\author{
BY \\ I. C. MICHAELSON \\ Haifa, Israel
}

IF a corneal graft is not properly coapted to its bed, a series of complications may ensue. These have been summarized by Barraquer (1950). A cause of this lack of coaptation is that the sides of the graft or of the bed may not be vertical, and that if sloping they may not slope in similar directions. A series of simple tests were therefore carried out to determine:

(a) The direction of the sides of the graft and how to influence it by the method of cutting the graft.

(b) The direction of the sides of the bed and how to influence it by the method of preparing the bed.

\section{Material}

Thirty-four human cadaver eyes were used. In each case the graft or bed was examined with the loupe and the dissecting microscope. Examination by means of the loupe is facilitated if the graft is placed on a blackened glass slide.

\section{Cutting the Graft (Fig. 1)}

(1) In thirteen eyes, 5-mm. grafts were cut with the cornea in position. The eye was held between the fingers, which exerted a firm but gentle pressure on the sides of the globe.

In nine cases the sides of the graft showed a slight convergence from the epithelial to the endothelial surfaces.

In three cases the sides of the graft were oblique and parallel.

In one case the sides of the graft were vertical.

(2) The same experiment was repeated with three other eyes, no pressure being exerted by the holding fingers on the sides of the globe.

In all three cases the sides of the graft showed a marked convergence from the epithelial to the endothelial surfaces.
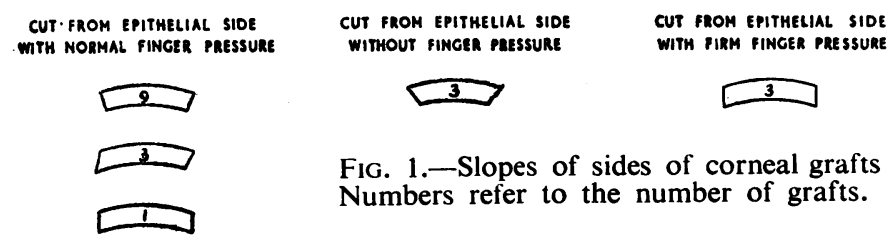

CUT FROA ENDOTHELIAL SIDE

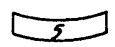

Fig. 1.-Slopes of sides of corneal grafts and recipient beds. Numbers refer to the number of grafts.

*Received for publication April 29, 1953. 
(3) The same experiment was repeated with three more eyes, a very firm pressure being exerted by the holding fingers on the sides of the globe.

In all three cases the sides of the graft were vertical.

In cases in which the intra-ocular pressure is not increased by the holding fingers, the cornea is dimpled by the trephine during cutting; in those cases where the intra-ocular pressure is increased by the holding fingers the cornea is not dimpled. It would appear, if the pressure of the trephine can produce a marked concavity of the corneal surface, that the graft edges converge inwards, the amount of the convergence being proportional to the concavity of the cornea.

(4) In five eyes the cornea was first removed and cut from the endothelial surface.

In all cases the sides of the graft were vertical.

The following conclusions may be drawn from these experiments:

(a) If the cut is made from the epithelial side of the cornea, the sides tend to be convergent.

(b) Pressure on the globe during cutting of the graft tends to make the sides vertical.

(c) If the cut is made from the epithelial side of the cornea, a definite proportion of grafts show parallel sloping sides.

(d) If the cut is made from the endothelial side of the cornea, the sides tend to be vertical:

It should be emphasized that the nature of the slope of the graft as described above, can be determined in an operating theatre if a loupe is used and the graft is placed on a black glass sjide.

\section{Preparing the Bed}

This experiment was carried out on ten eyes. The bed was prepared with the same care as used in the operating theatre. That is, the trephine incision was completed if necessary with scissors as soon as it became clear that the anterior chamber had been entered.

In three eyes it was practically unnecessary to use scissors. In each case the sides of the bed converged from the epithelial to the endothelial surface.

In seven eyes it was necessary to complete a good portion of the circumference of the bed with scissors.

In each case the side of the bed cut with the trephine showed a slope inwards (Fig. $2 a$ and $b$ ). The side cut with the scissors showed in its first portion a variable length sloping outwards and parallel to the side cut with the trephine. The portion of this side actually cut with the scissors showed a variable slope inwards.

\section{Discussion}

The sides of a corneal graft tend to be vertical if it is cut from the endothelial side or if it is cut from the epithelial side and a firm pressure is exerted by the holding fingers. Otherwise the sides tend to be convergent from the 


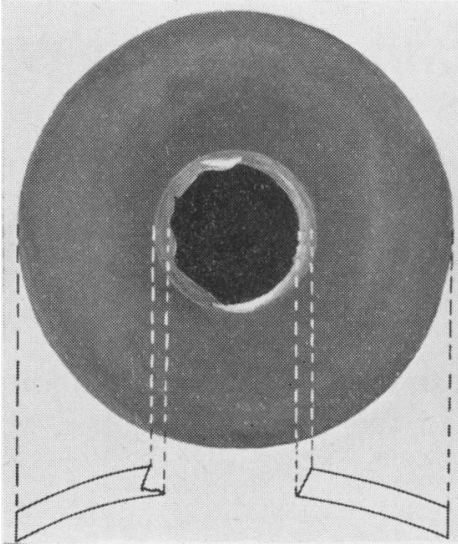

Fig. 2a.-Slopes of sides of recipient bed, and shape of disc removed.

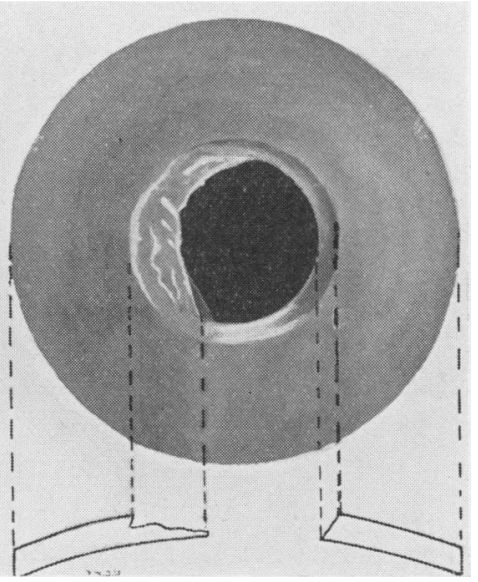

FIG. 2b.-Slopes of sides of recipient bed.

epithelial to the endothelial surfaces. In a definite proportion of cases, however, the sides of the graft are oblique and parallel. Such facts may have a clinical application in the operating theatre, and these possibilities are at present being investigated. For example, in one case where the sides of both graft and bed were oblique and parallel, the graft was orientated to conform to the slopes of the sides of the bed.

\section{Summary}

(1) The slope of the sides of a corneal graft cut with a trephine is shown to be vertical, convergent, or obliquely parallel.

(2) The factors influencing the direction of the slope are indicated. These include cutting from the epithelial surface, cutting from the endothelial surface, and intra-ocular pressure.

(3) The sides of the recipient bed are either convergent from the epithelial to the endothelial surfaces, or show parallel sloping surfaces modified by the projection of deep propria and endothelium which may result from the use of scissors.

(4) In cases where the sides of both the bed and the graft are sloping and parallel, the latter may be manoeuvred to conform with the sides of the bed.

This work was supported by a grant from the National Council to Combat Blindness, New York.

\section{REFERENCES}

Barraquer, J. I. (1950). Amer. J. Ophthal., 33, No. 3, pt. II, p. 6. 